\title{
Study of Brightness Preservation Histogram Equalization Techniques
}

\author{
Rasna $^{1}$, Vinod Karar ${ }^{2}$, Puneet Bansal ${ }^{3}$ \\ ${ }^{1,3}$ (University Institute of Engineering and Technology, Kurukshetra University, Kurukshetra, India) \\ 2(CSIR - Central Scientific Instruments Organisation, Chandigarh, India) \\ ('rasnakaswan@gmail.com, ${ }^{1}$ vkarar@ rediffmail.com, ${ }^{3}$ puneet4u@gmail.com)
}

\begin{abstract}
Image captured under various environment conditions with low contrast results in visual deterioration. To enhance the contrast of images a number of techniques have been proposed over the years. Histogram equalization is one of those methods which could be used for this purpose. For the contrast enhancement purpose histogram equalization (HE) attempts reduction in the number of gray levels. Contrast enhancement by histogram equalization method involve mapping of gray levels on the basis of changeover function that is derived from the probability distribution of gray levels embedded in input image. For this, the neighbouring gray levels in image having low probability density values are combined into single gray level value. Whereas for neighbouring gray levels having high probability density value the gap between them is increased, HE often makes over-enhancement of images with frequent gray level region resulting in loss of contrast for less frequent area of the images. Thus, the HE technique is not able to preserve image brightness. So wherever the preservation of image brightness is required this method is not preferred. This paper review various histogram equalization techniques used in preserving image brightness as well as the contrast enhancement is presented. The key approach for this aim is to use image segmentation. The criteria used are decomposition of images and the presentation of the basic difference between these techniques. For comparative study of performance of these techniques, absolute mean brightness error (AMBE) is used to determine degree of brightness preservation while peak signal to noise ratio (PSNR) have been used to fine $\backslash$ degree of contrast enhancement.
\end{abstract}

Keywords - Histogram Equalization, Brightness Preservation, Gray Level, Sub-image.

\section{INTRODUCTION}

Image enhancement is subjective arena of digital image processing. It is relevant for human illusion as well as computer vision. This aims at improving important and targeted features of the target image to fetch further details in the image. HE modifies intensity of original image such that the resultant image is made subjectively superior [1]. Image enhancement is a method to enhance the illustration and impression of information contained in images useful for analysis and provide adequate input to automatic image processing applications. It is not necessary that image enhancement technique which is adequate for one problem is also adequate for another (the result may be improvement or degradation in performance). Contrast changes the color or luminance impression due to which an object looks distinct. The information in any particular area of an image may be lost if contrast remains concentrated in particular region. The contrast enhancement thus forms an important step for image enhancement; however, the problem remains in preserving brightness of the image while image features are enhanced.

\section{DISCUSSIONS ON HISTOGRAM EQUALISATION TECHNIQUES}

Out of different techniques such as gray scale transformation, filtering and HE used for image enhancement, HE is preferred in most of the cases due to its simple implementation and effective delivery. HE reassigns the pixel intensity value such that we have a uniform intensity distribution. In this method contrast enhancement is performed by expanding the limits of gray levels. In addition it also flattens the histogram. However, there are few disadvantages associated with HE method. While it is not able to preserve the image brightness, it also causes the 'mean shift' which means that the mean intensity value is relocated to the intensity of the middle gray level of the image [2].

The Bi-histogram equalization works by separating the histogram of the input image in two sub-histograms. 
This is followed by equalizing every sub-histogram separately. Hence this method causes mean brightness of resultant image lie between input average level and middle gray level of the input image.

In brightness preserving histogram equalization (BPHE) method, the original image is separated in two subimages and mean gray level value $X_{m}$ is used as the separation intensity to decompose the image. While one sub-image contains set of samples which are less than or equal to mean value, the other sub-image has the set of samples, which is more than the mean value. This process is followed by HE operation on each of the two subimages independently. Hence, intensity values in the previous set of samples are mapped to intensity values varying from minimum gray level value to the input mean gray level values. Similarly, later set of samples are mapped to the range from mean gray level to the maximum gray level [3, 4].

Similar to the BPHE method, dualistic sub-image histogram equalization (DSIHE) divides initial image into two sub-images having equal area, that is, one dark and the other bright sub-image, but the division is carried considering median gray level. Then HE is applied on each of the two sub-images. The two processed subimages are then combined in a single image after equalizing operation on them. The brightness of resultant image is effectively the mean of equal and middle gray level of the image. No difference is found when the final image set is matched with the initial image $[3,5]$. The disintegration of the image is done with the aim that the Shannon's entropy is maximized. Under certain conditions due to significant luminance changes after equalization process, this process is not used in video system directly. This method yields maximum entropy for resultant image.

In the minimum mean brightness error bi-histogram equalization method (MMBEBHE), input image is separated in sub-images together. This is done so as to minimize brightness distinctness between the input and the output image decided considering the threshold value [3,7]. The parameter utilized in this is regarded as the absolute mean brightness error (AMBE). It is regarded as the difference between intensity value of each pixel of the initial image as well as the resultant image. The value of AMBE reduces when separation increases; hence lower value of AMBE is desired for brightness preservation. The major downside of this method in real time application is that large computation is required for the very first step of this method, especially when image consist of large number of gray levels.

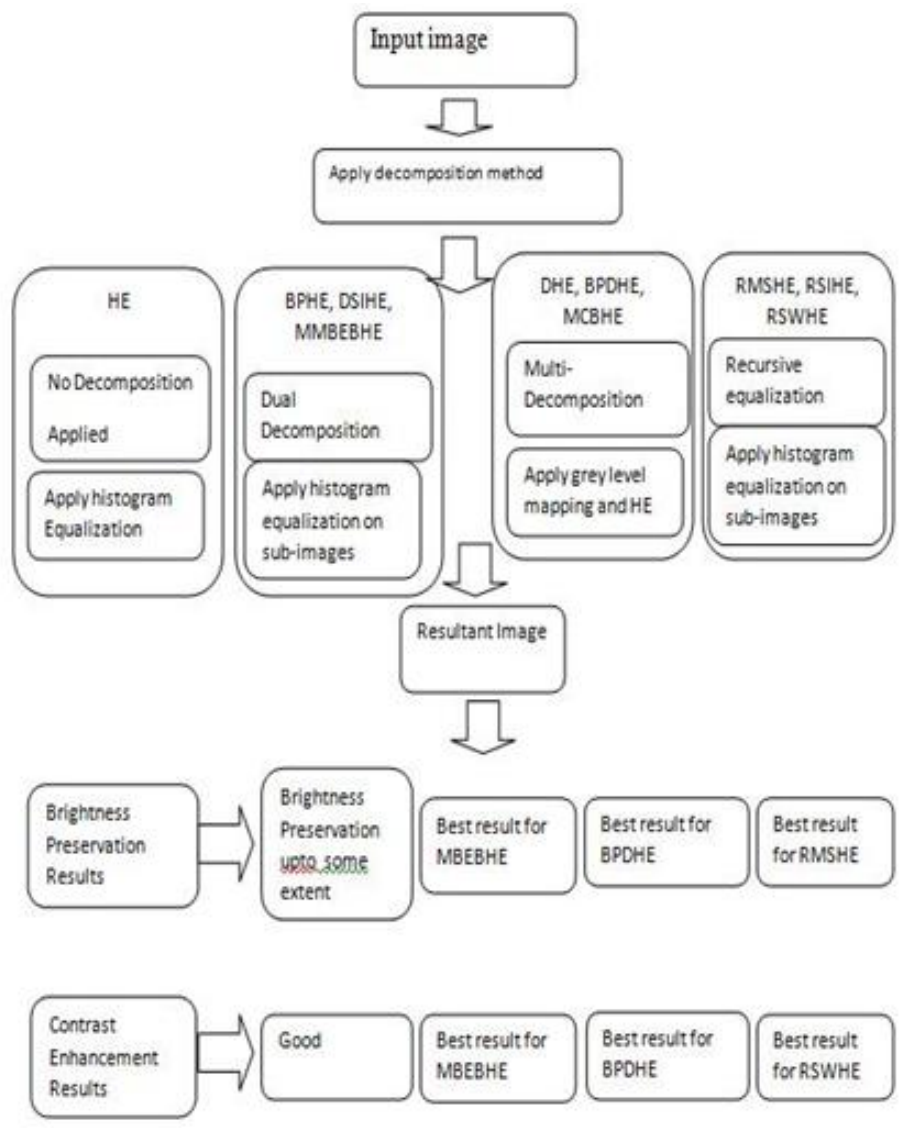

Fig 1: Broad flow chart for different histogram equalization methods 
Under the category of multiple level histogram equalization there are various techniques. In dynamic histogram equalization (DHE) the tendency of histogram equalization to produce undesired artefact is overcome by dynamic histogram equalization to enhance the image so that the input image is enhanced without the loss of important visual details [5,10]. The input image is separated in number of sub-images. This is done till no despot portion is there in any of the sub-image. For mapping of gray-level of sub-image by HE a dynamic graylevel is designated to them. This designation process is based on dynamic range of each sub-image and also CDF of the histogram values [5]. Thus wash-out and overshadow problem of small features of input image is prevented. The brightness preserving dynamic histogram equalization method (BPDHE) is addition on MPHEBP and DHE to solve the issue of mean shift. The output image provided by this method has the same mean intensity as there in the input image. As in MPHEBP the input image histogram is smoothened and the separation of the histogram is carried out based on the local maxima of smoothed histogram [9]. Each subhistogram is mapped to new dynamic range before histogram equalization is applied. As mean brightness changes with change in dynamic range normalization of output intensity is done at the last stage.The multilevel component based histogram equalization (MCBHE) method is an addition to BPBHE method. It enhances the global as well as the local image contrast with less distortion. The variation from the brightness preserving based histogram equalization (BPBHE) is that the BPBHE uses traditional HE method for equalizing each subimage while MCBHE uses multiple gray level thresholding in addition to connected components for sub-image equalization. A predetermined gray level is used for thresholding purpose. To recognize the connected component (below or above current threshold) labelling is performed [10]. Main advantage is that the variations of gray level are captured with change in threshold value [11].

The recursive mean separate histogram equalization (RMSHE) is an extension of traditional HE and BPHE which employs mean of the input image to decompose the image. While BBHE decompose the input histogram just once, RMSHE method carry out decomposition of input image continuously up to a level ' $R$ ' resulting in $2 \mathrm{R}$ sub-images. The HE technique is performed for each sub-image separately if $\mathrm{R}=0$ which means that no mean separation is done before $\mathrm{HE}$, and then this method is equivalent to HE only. If $\mathrm{R}=1$ then sub-image decomposition is done once. The brightness preservation is better in this case as value of $\mathrm{R}$ increases as shown in [12]. Main disadvantage of this method is that the number of decomposed sub-histogram is equal to power of 2. The recursive sub-image histogram equalization is comparable to RMSHE with only difference in the condition that it uses median for decomposition purpose, similarly, the recursive separated and weighted histogram equalisation technique (RSWHE) modifies the input histogram. It employs three modules [13]. Histogram segmentation module decomposes the input histogram into two or more sub-histograms based on the mean and the median value $[14,15]$. Modification of sub-histogram through histogram weighting process that uses power law function is done in the second module. Subsequently, histogram equalization is applied for the modified and weight sub histogram.Broad flow chart for various histogram equalization methods is shown in Fig. 1.

\section{MERIT PARAMETERS FOR COMPARASION}

Various parameters used to assess the performance of different HE techniques in preserving image brightness and also enhance the contrast, are presented in this section. Preservation of brightness by different method is compared on the basis of AMBE while the image quality is characterized by PSNR.

The PSNR is defined as the ratio of the maximum feasible power level of the signal to the power of the noise which affects fidelity of its nature. It is utilized to evaluate quality of the reconstructed image. The PSNR is represented as:

$$
\mathrm{PSNR}=10 \log \left(255^{2} / \mathrm{MSE}\right)
$$

Where, 255 is maximum value attained by any image signal and MSE is the mean square error between decompressed and original image.

The AMBE, which is the difference between average intensity of initial image and resultant image, has lower value where brightness preservation is to be achieved. It is represented as: 


$$
\mathrm{AMBE}=\mathrm{E}(\mathrm{x})-\mathrm{E}(\mathrm{y})
$$

The Table 1 provides comparison of various histogram equalization techniques.

Table I Comparison of different histogram equalization techniques [16]

\begin{tabular}{|c|c|c|c|c|}
\hline $\begin{array}{l}\text { Sr. } \\
\text { No. }\end{array}$ & $\begin{array}{c}\text { Contrast } \\
\text { Enhancement } \\
\text { Method }\end{array}$ & Process Followed & $\begin{array}{l}\text { Absolute Mean } \\
\text { Brightness Error }\end{array}$ & Remarks \\
\hline 1. & $\mathrm{HE}$ & Calculate distribution function & $\begin{array}{l}\text { Minimum up to } \\
\text { some extent }\end{array}$ & Good \\
\hline 2. & BPHE & Find out mean of the input image & $\begin{array}{l}\text { Minimum as } \\
\text { compared to } \mathrm{HE}\end{array}$ & Better than HE \\
\hline 3. & DISHE & $\begin{array}{l}\text { Decomposition based on median } \\
\text { of the original image }\end{array}$ & $\begin{array}{l}\text { Almost same as } \\
\text { BPHE }\end{array}$ & $\begin{array}{l}\text { Almost same as } \\
\text { BPHE }\end{array}$ \\
\hline 4. & MMBEBHE & $\begin{array}{l}\text { Least value of brightness } \\
\text { difference between initial and } \\
\text { resultant image is computed }\end{array}$ & Less than DSIHE & $\begin{array}{l}\text { Much better than } \\
\text { DSIHE }\end{array}$ \\
\hline 5. & DHE & $\begin{array}{l}\text { Decomposition of image until } \\
\text { dominating portion of the image is } \\
\text { eliminated }\end{array}$ & Poor & $\begin{array}{l}\text { Poor contrast } \\
\text { enhancement }\end{array}$ \\
\hline 6. & BPDHE & $\begin{array}{l}\text { Attempt to preserve mean intensity } \\
\text { of the image }\end{array}$ & $\begin{array}{l}\text { Improved than } \\
\text { others }\end{array}$ & $\begin{array}{l}\text { More improved } \\
\text { than others }\end{array}$ \\
\hline 7. & MCBHHE & $\begin{array}{l}\text { Employ multi-gray level threshold } \\
\text { and connected component analysis }\end{array}$ & Better than HE & $\begin{array}{l}\text { Both local and } \\
\text { global contrast } \\
\text { enhancement }\end{array}$ \\
\hline 8. & RMSHE & $\begin{array}{c}\text { Decomposition of image is carried } \\
\text { out recursively using mean of } \\
\text { input image }\end{array}$ & $\begin{array}{l}\text { Better improvement } \\
\text { of the dark images }\end{array}$ & Over-enhance noise \\
\hline 9. & RSIHE & $\begin{array}{l}\text { Recursive decomposition of image } \\
\text { using median value }\end{array}$ & Better than RMSHE & Good \\
\hline 10 . & RSWHE & Use histogram weighing function & $\begin{array}{l}\text { Poor brightness } \\
\text { preservation }\end{array}$ & $\begin{array}{l}\text { Better contrast } \\
\text { enhancements }\end{array}$ \\
\hline
\end{tabular}

\section{CONCLUSION}

A comparative study of various brightness preserving histogram equalization techniques was carried out. BPHE and DSIHE are able to preserve mean intensity of the image. It also results in enhancement of the image contrast. In MMBEBHE brightness preservation is found to be improved, however, more distortion is observed. The DHE results in superior performance than MMBEBHE having reduced distortion. Brightness preservation is better in BPDHE than all the other methods. MCBHE enhances both global and local contrast of the image. 


\section{REFERENCES}

[1] Kotkar V. A. And Gharde S. S., "review of various image contrast enhancement techniques", International Journal of Innovative Research in Science, Engineering and Technology, Vol. 2, Iss. 7, July 2013.

[2] Sonam, Rajiv Dahiya, "review of contrast enhancement techniques based on histogram equalization", International Journal of Advance Research in Education Technology (IJARET), Vol.2, Iss. 2, April-June 2015.

[3] Manpreet Kaur, Jasdeep Kaur, Jappreet Kaur., "Study of image enhancement techniques: a review", International Journal of Advanced Research in Computer Science and Software Engineering, Vol. 3, Iss. 4, April 2013, ISSN: 2277 128X.

[4] Kim Y. T., "Contrast enhancement using brightness preserving bi-histogram equalization", IEEE Trans. Consumer Electronics, vol. 43, No. 1, pp. 1-8, Feb. 1997.

[5] Shefali Gupta, Yadwinder Kaur, "Review of different histogram equalization based contrast enhancement techniques", International Journal of Advanced Research in Computer and Communication Engineering, Vol. 3, Iss. 7, July 2014

[6] Y. Wang, Q. Chen, and B. Zhang, "image enhancement based on equal area dualistic sub-image histogram equalization method", IEEE Trans. on Consumer Electronics, Vol. 45, No. 1, Pp. 68-75, Feb. 1999.

[7] S. Der Chen and A. R. Ramli, "Minimum mean brightness error bi-histogram equalization in contrast enhancement," IEEE Trans. Consum. Electron., vol. 49, no. 4, pp. 1310-1319, 2003.

[8] M. Abdullah-Al-Wadud, M. Kabir, M. Akber Dewan, and O. Chae, "A Dynamic Histogram Equalization for Image Contrast Enhancement," IEEE Trans. Consum. Electron., vol. 53, no. 2, pp. 593-600, 2007.

[9] Haidi Ibrahim, and Nicholas Sia Pik Kong, "brightness preserving dynamic histogram equalization for image contrast enhancement", IEEE Trans. Consumer Electronics, Vol. 53, No. 4, pp. 1752 - 1758, November 2007.

[10] Jafar, H. Ying, "Multilevel component-based histogram equalization for enhancing the quality of gray scale images", Electro/Information Technology, 2007 IEEE International Conference on, pp. 563-568.

[11] Aniketa Agrawal, Atish Mishra, and Praveen Bhanodia, "A comparative study of widely used histogram equalization based image contrast enhancement methods", International Journal of Advanced Research in Computer Science and Software Engineering, Vol. 4, Iss. 12, December 2014.

[12] S. D. Chen, A. Ramli, "Contrast enhancement using recursive mean separate histogram equalization for scalable brightness preservation", IEEE Trans. on Consumer Electronics, Vol. 49, No. 4, pp. 1301-1309, Nov. 2003.

[13] M. Kim, and M. G. Chung, "Recursively separated and weighted histogram equalization for brightness preservation and contrast enhancement", IEEE Transactions on Consumer Electronics, Vol. 54, No. 3, August 2008.

[14] Patel O., Yogendra P. S. Maravi, and Sharma S., "A comparative study of histogram equalization based image enhancement techniques for brightness preservation and contrast enhancement", Signal \& Image Processing: An International Journal (SIPIJ) Vol. 4, No.5, October 2013

[15] Wang Yuanji. Li Jianhua, Lu E, Fu Yao, and Jiang Qinzhong, "Image quality evaluation based on image weighted separating block peak signal to noise ratio", IEEE Int. Conf. Neural Networks \& Signal Processing, Nanjing, China, December 14-17, 2003.

[16] Chhaya Gautam, and Neeraj Tiwari "A comparative study of histogram equalization techniques and adaptive gamma correction for color image contrast enhancement", International Journal of Computer Applications, ISSN (0975-8887, Vol. 103, No. 15, October 2014. 